\title{
DETERMINATION OF CIGARETTE SMOKE DISPERSION USING OPTICAL RGB CAMERA AND DIGITAL IMAGE PROCESSING
}

\author{
J. Y. Bernardino ${ }^{1}$, K. N. Velasco ${ }^{1}$, R. V. Ramos ${ }^{1}$, K. P. Vergara ${ }^{1}$ \\ ${ }^{1}$ Department of Geodetic Engineering, University of the Philippines - Diliman, Quezon City, Philippines - (jybernardino, \\ knvelasco, rvramos, kpvergara)@up.edu.ph
}

Commission IV

KEY WORDS: Cigarette Smoke, Smoke Dispersion, Optical RGB Camera, Digital Image Processing, Visualization

\begin{abstract}
:
Across ages, the rapid prevalence of cigarette smoking has consistently posed adverse threats to both human health and the environment. Moreover, smoke dispersed from a single cigarette could expose serious respiratory problems to a number of non-smokers within the area. Cigarette smoke has always been a notorious, human-induced health risk and air pollutant thus, the urgent need to develop smoke management schemes by exploring strategies on observing cigarette smoke dispersion. This research aims to incorporate the potential of optical RGB cameras in the study of smoke dispersion. Through digital image processing of experimental smoking videos, a spatiotemporal visualization of smoke dispersion for an indoor and outdoor environment were created. Smoke movement starting from the source was observed in terms of smoke pixel density and maximum horizontal extent. Quantitatively, the results showed a relative maximum extent of 1.21 meters which lasted for 2 seconds for an outdoor environment while 1.05 meters which lasted for 6 seconds for an indoor environment. The maximum relative smoke pixel density values calculated for the outdoor and indoor environment are $1.46 \%$ and $1.12 \%$ respectively. The resulting graphs were indicative of a trend that creates a normal distribution curve that can be affected by external factors and represent a function relating dispersion and distance. The results of this study prove the capability of optical RGB cameras as an alternative and cost-efficient method in studying smoke dispersion. Furthermore, this practical method of monitoring smoke dispersion could lead to comprehensive analyses of air quality management and health exposure assessments.
\end{abstract}

\section{INTRODUCTION}

\subsection{Background of the Study}

Air pollution has always been an alarming environmental problem and a health concern to the public. Air pollution sources and atmospheric processes that form, transport, and remove ozone, and precursor species determine when and where air quality problems will occur. In many types of research, not identifying the specific air pollution source can lead to continued threats to the health of the public (Lu et al., 2018).

On the contrary, air pollution is being produced by numerous factors, for instance, cigarette smoke contributes 10 times greater than a diesel car exhaust to the air pollution according to an article by an online medical newsletter. In line with these studies, there is an urgent need not only to raise awareness but to explore how to protect public health and create strategies for controlling cigarette smoke. There have been a lot of studies on how Geographic Information System (GIS) and Remote Sensing (RS) can help with air quality assessment and give a comprehensive analysis of air quality management, though the usage of Photogrammetry is yet to be explored.

Close-Range Photogrammetry (CRP) is a category under photogrammetry that uses images and range data of a subject less than 1,000 feet away to the instrument that collects images (Salmon, 2014) to extract reliable geometric and thematic information. An example of a set-up that shows $\mathrm{CRP}$ is using a camera positioned in a specific distance from the subject to acquire images. In photogrammetry, a wide range of software can be used for the processing and analysis of images. These processes and analysis that may be done shall include applying photogrammetric techniques to eliminate error acquired from images, to transform all imagery measurements to real-world measurements and lastly, to determine different factors that may or may not affect the subject. This study will focus on the feasibility of CRP in air quality management through determination of smoke dispersion, specifically cigarette smoke, and the creation of a methodology that can map out cigarette smoke extent and movement in two (2) different environments: indoor and outdoor. This study will also try to test the usage of an optical RGB camera for cigarette smoke detection.

This study will focus on the feasibility of CRP in air quality management through determination of smoke dispersion, specifically cigarette smoke, and the creation of a methodology that can map out cigarette smoke extent and movement in two (2) different environments: indoor and outdoor. This study will also try to test the usage of an optical RGB camera for cigarette smoke detection.

\subsection{Research Objectives}

There are two main objectives that this research aims to fulfill: (1) to test the feasibility of optical RGB camera as the main instrument for the determination of cigarette smoke dispersion, specifically, using various photogrammetric software and digital image processing techniques and (2) to create a spatiotemporal visualization of the smoke dispersion through a video output from an executable file. The visual representation of the cigarette smoke would be used to determine its relative maximum horizontal extent and its relative smoke density quantitatively. 


\subsection{Significance of the Study}

The protection of public health does not only constitute raising awareness but also creating new strategies for controlling cigarette smoke. A lot of researches and journal papers focus on how GIS and RS were utilized to create comprehensive analysis on air quality management, but the use of photogrammetry and its application on digital image processing to aid in air quality assessment and management is yet to be explored. This study may provide an alternative cost-efficient method in studying smoke dispersion through an approach by using an optical RGB camera as the main instrument. The findings of this study could be useful in proposing safe zones or a buffer distance of a smoking area adjacent to sidewalks or pedestrian walkways. The results of the study will be used to provide information and raise awareness to the public on how a cigarette smoke disperse through an environment over time. Also, the methods in the study may be adopted and could be used as a low-cost alternative for health exposure assessment in medical researches.

\subsection{Scope and Limitations}

The indoor environment utilized in the study is inside a living room of an apartment that was enclosed and with a dimension of $3 \times 3$ meters (Refer to Figure 1). It was assumed that the enclosed study area is not affected by external factors such as wind. The assumption of absence of wind was not measured using any sensors but logically presumed because all the possible sources of external factors were controlled. The selected research study area for our outdoor environment is in an open space parking lot in between the 2 buildings located in UP Diliman, Quezon City (Refer to Figure 1). It was assumed to be affected by ambient wind and temperature. The assumption on the presence of different external factors were again not measured by any sensors. The acquisition of data at the indoor environment were during low wind and humid conditions, while there is fair amount of sun and wind at the outdoor environment. With the given atmospheric conditions, these differences are not considered to affect the results of the imaging process.
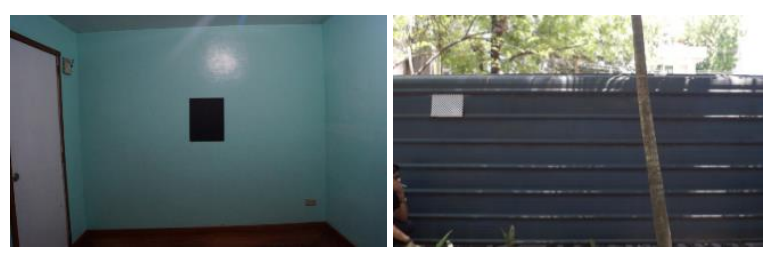

Figure 1. Study area for indoor environment (left) and outdoor environment (right)

In the visualization of the cigarette smoke dispersion, the analysis was limited to the two-dimensional aspect (i.e. along the plane normal to the ground). The analysis with the threedimensional visualization of a smoke dispersion would be very tedious with its camera set-up, in which there are synchronized multiple camera in different angles to ensure overlap and to create a simple stitched image. This complicated set-up is not cost effective. On the other hand, keeping the smoke in its two-dimensional aspect will remove the uncertainty of the smoke moving toward or away the camera and having thickness.

The term smoke density is associated with its opacity measurement such as optical depth and haze percentage, thus the usage of the term in this study, smoke density relating to the covered pixels of the smoke over a frame. The precision of encapsulating the relative smoke density of the smoke and maximum horizontal extent in a frame, was limited to the process of data acquisition and processing using the software. The processing time and the quality of the resulting outputs such as the images were constrained by the capacity and the performance of the computer used. The study will focus in the analysis of the smoke's relative density and horizontal extent.

\subsection{Review of Related Literature}

Cigarette contains various harmful chemicals and once consumed through smoking, releases pollutants that greatly contribute to air pollution. Pollutants are transferred from the air to another medium or object through the process of dispersion which is also called transmission (Baumbach, 2014). Generally, characterizing smoke in an open environment involves various methods and solutions. Due to external forces, the dispersion of air contaminant can be quickly affected by number of factors and thus become unpredictable (Lorenz, 1963). Also, every characterization of the dispersion will be somewhat different (Wyngaard, 2010). Hence, the consistent effort to study this topic. There have been a lot of studies of smoke dispersion using Remote Sensing (RS) and Geographic Information System (GIS) but with the rapid developments in digital camera technology, photogrammetric methods can now be explored to study smoke. (Haupt et al., 2014)

Studies stated that the integration of smoke detection in photogrammetric techniques was derived from the intention of generating an algorithm for fire detection (Celik et al., 2007). The use of optical camera and photogrammetric techniques in smoke detection and determination became visible through time. Vision-based methods are now also used by researches for smoke detection and algorithms can be categorized into three groups. It can be by motion-based, appearance-based, or energy-based method. (Wang et al., 2012) Motion detection in a video can be acquired by subtracting two successive frames. The resulting image which is the difference of two successive images is binarized by comparing pixel values. Equation 1 gives the mathematical aspect of image subtraction wherein $d$ is the resulting image and $t$ is the frame for an instantaneous time.

$$
d(x, y, t+\Delta t)= \begin{cases}1 & \text { if }|I(x, y, t+\Delta t)-I(x, y, t)|>T_{1} \\ 0 & \text { otherwise }\end{cases}
$$

Other methods for smoke detection were introduced by different studies such as image accumulation to combine subtracted images, image binarization to remove noises, and morphological operations to clean the images (Deldjoo et al., 2015). Appearance-based method used shape, color, texture, etc. to detect smoke. (Wang et al., 2012) Smoke color, in the analysis of smoke object, is obtained by the condition that $\mathrm{R}$ $\pm \mathrm{a}=\mathrm{G} \pm \mathrm{a}=\mathrm{B} \pm \mathrm{a}$ in RGB color space. It implies that the three components of smoke pixels are equal and can be used in smoke pixel determination. (Mutar, Dway, 2018) Smoke's appearance in vision-based detection can be also treated as an object. Open and close filter is used to partition object. To remove noise pixels that has smoke features without sacrificing big data losses, open filter is used. On the other hand, close filter joined divided smoke pixel. (Hwang, Jeong, 2016) Energy based method used wavelet energy to study smoke behaviour. 
In line with the various features of smoke that can be used for image processing, OpenCV is one of the useful libraries that can be used in generating vision-based smoke detection program. It runs under different operating systems and provides interfaces for various languages. The library contains abundant and useful math functions, image processing functions, and computer vision functions. It also includes different modules with $\mathrm{CV}$ module as the main module that can be used in image processing, structure analysis, motion analysis, object tracking, and a lot more.

Smoke dispersion has a lot of characteristics or property that can be quantitatively analysed using different photogrammetric techniques. A study by Sue Ellen Haupt entitled Applying Photogrammetric Techniques to study Smoke Plumes suggested the use of a checkered board background with known measurements to get the optical depth and opacity of the smoke plume (Haupt, 2014). But having a known measurement of an object in an image, using photogrammetric techniques, relative measurements of any sort can be acquired. This concept may be used in obtaining one of the characteristics of smoke dispersion which is its extent. Another property of smoke that is visible and recognized by the human eye is the smoke density. It can be defined as the decrease of background details caused by the smoke. It is not homogenous and varies in different regions in an image. From this statement, it can also be defined as the relative area occupied by the smoke in an image. This is useful in analysis of smoke's exposure in certain region or location of an image. Both properties, smoke extent and smoke density, can be helpful in studying the relative behavior of the smoke dispersion in certain conditions.

There is a wide number of air dispersion models. In the case of smoke, dispersion or distribution may be treated as puffs or series of puffs also called as plume. The most common representation of a plume is if the movement is treated at an averaged time interval. The graph of the smoke extent relative to time resembles a normal curve based from different studies. This is if it takes into account the effect of a constant wind speed and wind direction in space and time relative from the source. (Visscher, 2014)

\section{METHODOLOGY}

There are three general steps for the methodology, mainly, data gathering, data processing and lastly, data analysis.

Data gathering involve acquisition of videos in the two different environment- outdoor and indoor environments. For the indoor environment, a large checkerboard was set as the background not only to be a marker for the measurement but to emphasize the color of the smoke. For the outdoor environment, a piece of this checkerboard was set at the background to be a marker. The videos acquired were then used to input in Adobe Premiere Pro to undergo splicing. Splicing the videos resulted to a single puff video wherein at $\mathrm{t}=0$ was when the smoke started to leave the source. This would be an easier used for data processing and data analysis.

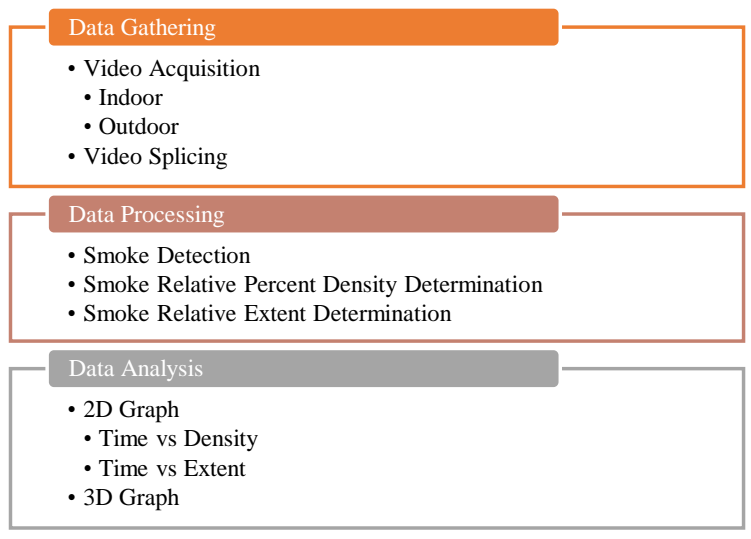

Figure 2. General Flowchart for the Methodology

The single puff video was used as an input in the generated code wherein OpenCV and Numpy library was used. This video would then undergo Gaussian blurring to remove unwanted noise. InRange function would then be applied to the video. InRange function takes an upper bound limit and lower bound limit, since it was known that the smoke color was ranging from light gray to dark gray color, it is used to locate smoke pixels. After this, Image Subtraction to detect motion was then used. The specific background subtractor function used was BackgroundSubtractorMOG2 (Mixed of Gaussian) for it can also detect shadows. Shadow can be a possible smoke pixel since it also shares the same characteristic. Morphological Transformations, specifically closing filter which was used for closing up small holes inside the object detected and opening filter for removing unwanted pixels was used respectively. After this, thresholding to remove shadow pixels was applied to get the binarized smoke detected.

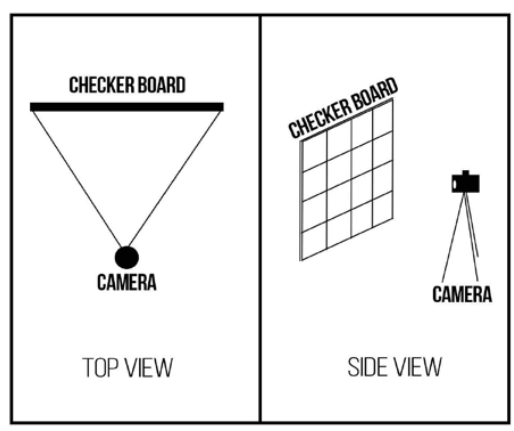

Figure 3. Illustration of the Camera Set-up

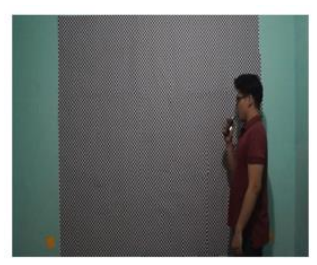

Indoor Set-Up

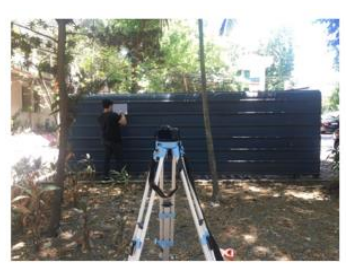

Outdoor Set-Up
Figure 4. Environment Set-up 

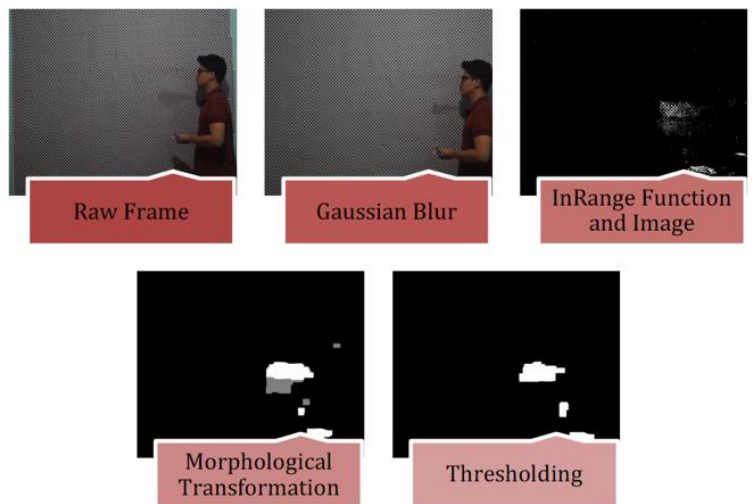

Figure 5. OpenCV Functions Applied to a Sample Frame

This resulting binarized smoke detected video was also used in masking the raw video to have an output wherein the smoke was highlighted in the raw video. Drawing contour function was also used to return a video that could enclose the smoke pixels in a bounding rectangle that later used in computation for the extent. Different variations of the output video were saved to help in visualization and analyzation.

Smoke Percent Density $=\frac{\text { Number of Smoke Pixels }}{\text { Total Number of Frame Pixels }}$

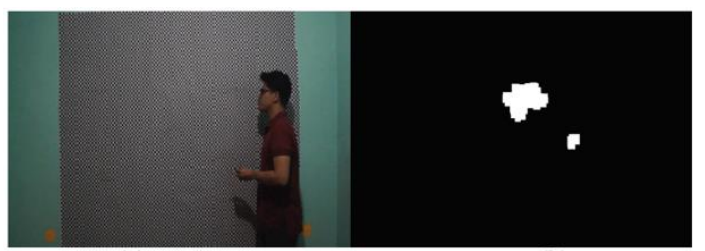

(a) (b)

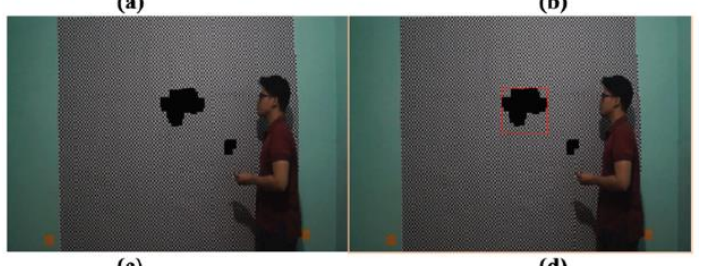

(c)

(d)

Figure 6. Variations of Output Video for Analyzation (a) raw video frame (b) binarized video frame (c) masked video frame (d) the masked video with contour frame

The smoke detected video with contour output, that extracted the smoke from all the other elements on the frame, was processed using Adobe Premiere Pro to extract frames in a 0.4 second interval. The frames acquired was added to ArcMap to transform the data from raster to vector data using the Raster to Polygon tool under Spatial Analyst Toolbox. The tool automatically creates an attribute table containing all the spatial data needed like frame area and grid code for the cigarette smoke. Using the editing tool, each frame with smoke was processed using the polygon tool and capture the area of the smoke in a frame. These values were divided by the total area covered by the whole frame then be converted to a percentage resulting in the percentage density value obtained for each frame shown in Equation 2 that was in a 0.4 -second interval. The obtained relative smoke density values were tabulated and was subject to analysis further on.

$$
\frac{\text { Known Ground Measurement }}{\text { Known Image Distance }}=\frac{\text { Smoke Extent }}{\left|X_{\text {Source }}-X_{\text {MaxExtent }}\right|}
$$

The resulting video with the bounding rectangle that detected the smoke was used to extract frame in a 0.4-second interval. Another program was created wherein the image coordinates of a specific image can be extracted. The pixel coordinate of the source acquired for the first step. This would act as the origin of the smoke. Frames were analysed to get certain coordinates of the maximum relative extent of the cigarette smoke. Knowing that a length of 8 pixels for indoors and 7 pixels for outdoors corresponds to 0.017 meters in real-world measurement, the real-world extent of the smoke relative to the origin can be computed by subtracting the maximum $\mathrm{x}$ coordinate of the cigarette smoke extent to the source's $\mathrm{x}$ coordinate and multiplying it with the conversion values derived from knowing the corresponding pixel length and real-world measurement shown in Equation 3. Instantaneous distances can be acquired and used in data analysis.
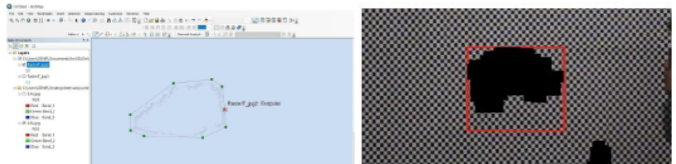

Figure 7. Frame Converted from Raster to Vector using ArcMap (left) and Frame wherein the Smoke Extent was Highlighted (right)

For the data analysis and interpretation of results, MS Excel was used to generate a 2D graph of the instantaneous cigarette smoke percent density and instantaneous cigarette smoke extent both in an indoor and outdoor environment. The graph was analyzed and interpreted using the related studies discussed. A 3D graph correlating the instantaneous smoke percent density and instantaneous cigarette smoke extent was also generated using PyCharm and the MatPlotLib library. For better analysis and visualization, different variations of the resulting video were compared side by side.

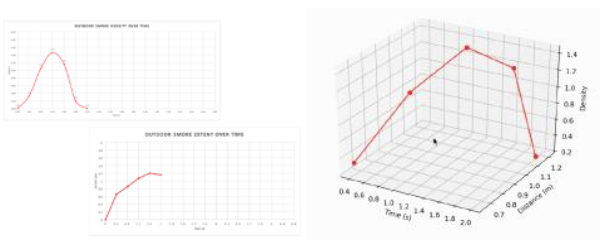

Figure 8. Example of the Generated Graphs in 2D (left) and 3D (right)

\section{RESULTS AND DISCUSSION}

\subsection{Indoor Environment Results}

Different options offered by executable file for this video were acquired to help in analyzation and visualization Figure 8 shows these different versions of output video. The first image was the 10th frame from the input video or the raw video while the second image was the binarized version of this frame generated after running the program. For better visualization, the third image which was the masked version 
of the raw video in which the binarized output video was overlaid to the raw video. The fourth image was the output video in which a red rectangle was drawn to delineate the smoke pixels from other moving pixels.
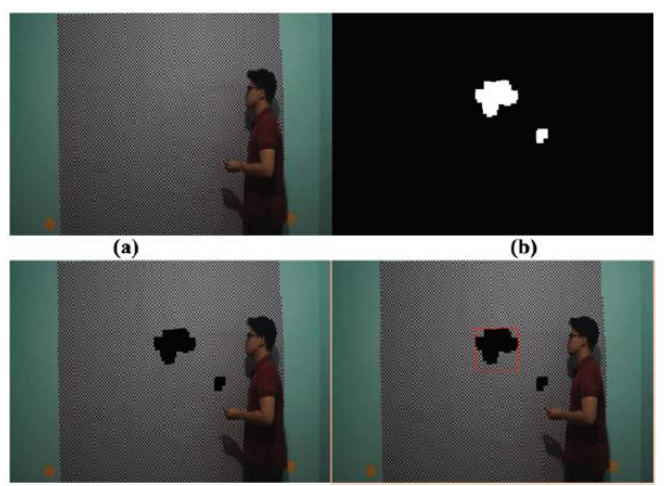

(c)

Figure 9. Peak Density of Cigarette Smoke in an Indoor Environment at 2.8 seconds ( $70^{\text {th }}$ Frame) (a) raw video frame (b) binarized video frame (c) masked video frame (d) the masked

The output video was extracted at 25 frames per second meaning that each frame corresponds to 0.04 seconds. It was observed that at 0.4 seconds or at the 10th frame of the extracted output video the cigarette smoke started to appear and at 6.32 seconds or at the $158^{\text {th }}$ frame the smoke started to not be detectable. On the other hand, the relative smoke density or the size of the smoke relative to the frame was at highest at 2.8- second mark of the output video or at 70th frame of the extracted frames from the output video as shown in Figure 9. These extracted frames were helpful in the analysis of the general behaviour in terms of density of the cigarette smoke in an indoor environment.

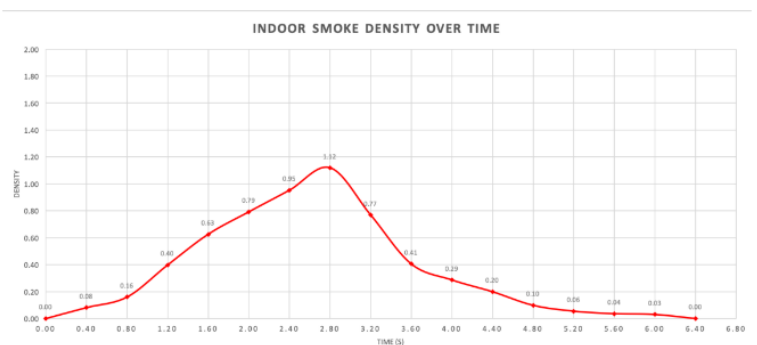

Figure 10. 2D Graph of Cigarette Smoke Density vs Time in an Indoor Environment

The percent density value at 2.80 seconds, the highest value achieved by the curve, was $1.119 \%$. The percent density then diminished drastically as the peak was achieved then the last smoke detected was at 6.32 seconds then dropped to 0 percent density at 6.40 seconds.

Besides relative density of smoke over time, another measurement derived from the extracted frames was the relative horizontal extent of smoke from the source over time. This was acquired using a known relationship between image distance and a real-world measurement distance.

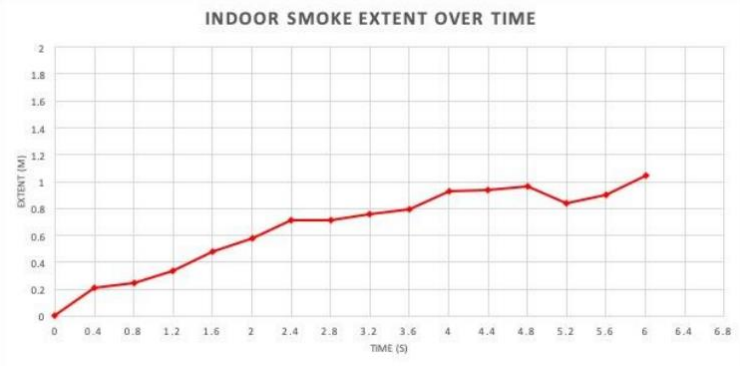

Figure 11. 2D Graph of Cigarette Smoke Extent vs Time in an Indoor Environment

The results shown in Figure 11 was that, at the 6.0-second mark of the output video, the smoke ended at exactly 1.05 meters. Observing the data, the smoke gradually increases its distance cover, yet at the interval of 4.8 seconds to 5.2 seconds the distance went down meaning that the smoke backtracked then went away again to its meant direction ending at 6 seconds. The resulting backtrack was an inconsistency on the detection done by the program since it does not account the transparent smoke, and knowing that at time of 5.2 seconds, the percent density of the smoke is nearing zero; thus, at this time the cigarette smoke's visibility was near disappearing and almost transparent resulting in a fluctuation on the contour on the smoke. The backtrack could also be a result of the imperfection of the study area for the indoor environment. Since the environment was assumed free from external factors like wind, it can also be a source of inconsistency because a small amount of wind could have affected the smoke. Wind sources penetrating to the small openings of the door facing against the movement of the smoke could be one of the candidates that caused the backtrack of the smoke.

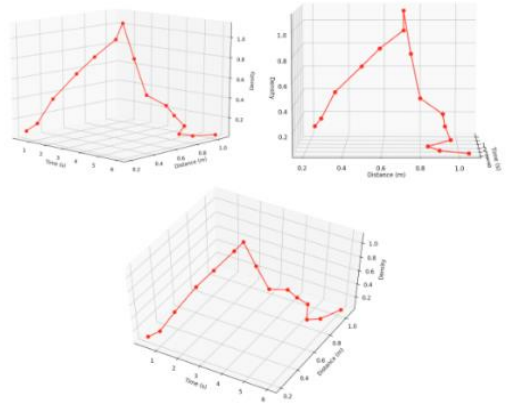

Figure 12. 3D Graph of Cigarette Smoke Density vs Time vs Extent Relative from the Source in an Indoor Environment

\subsection{Outdoor Environment Results}

A two-second outdoor video was also used as an input to the executable file. Same with what discussed in the methodology, different output videos were acquired to study the characteristic of cigarette smoke in an outdoor environment. Other external factors that were limited in an indoor environment was present in this condition such as wind factor and other visible objects that can affect how the smoke appeared. The output videos were extracted 25 frames per second being consistent with the previous method. 

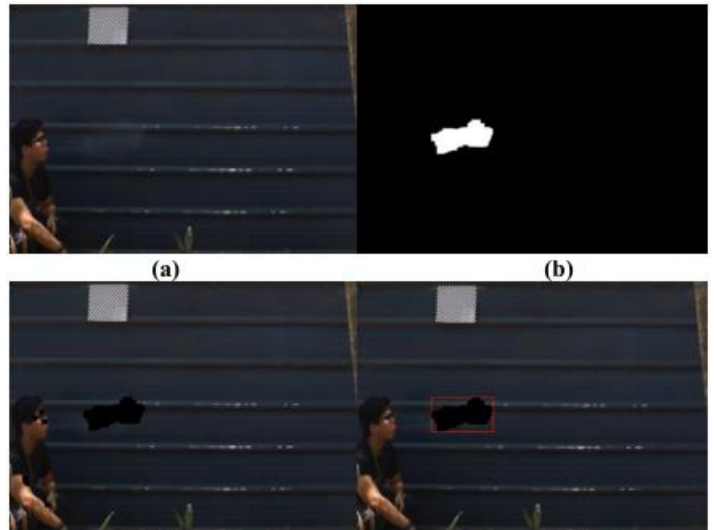

(c)

(d)

Figure 13. Peak Density of Cigarette Smoke in an Outdoor Environment at 1.2 seconds (30th Frame) (a) raw video frame (b) binarized video frame (c) masked video frame (d) the masked video with contour frame

The extracted frames from the output videos were then analyzed to get the relative density of the cigarette smoke and its extent relative to the source. A 0.40 -second interval, same with the indoor environment, was set in acquiring data to have general information of the characteristic of the cigarette smoke.

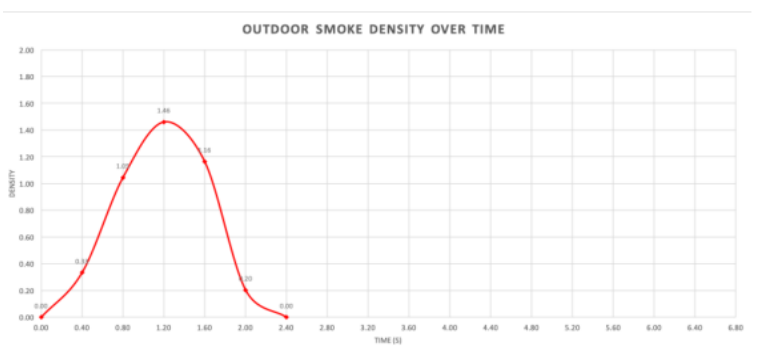

Figure 14. 2D Graph of Cigarette Smoke Density vs Time in an Outdoor Environment

Using the acquired relative smoke density of the cigarette smoke in each corresponding time, a $2 \mathrm{D}$ graph of the cigarette smoke density vs time in an outdoor environment was created. The graph as shown in Figure 14 shows that as time progresses, the smoke pixel present in each frame also changes. It shows that the smoke pixel density was at its highest value and from the figures shown above it was found that the percent density at the mark was $1.46 \%$. The graph forms a curve in which the percent density increases as the time increases and after reaching the highest point the percent density decreases until there was none. It was in the same behaviour with the indoor environment that explains how smoke, when puffed out of mouth takes up a large area of the environment, diminishes through time even when affected by different external factors.

The extent of the smoke relative to the source as shown in Figure 15 was also measured using the image's x-coordinate of the source that was also acquired from the created program, this $\mathrm{x}$-coordinate would be the origin or the relative origin of the smoke to derive its extent. A marker with known real measurement was placed at the environment. It was found, using this marker, which was measured 7 pixels from the image have a real-world measurement of 0.017 meters and later used in acquiring the relative extent of the cigarette smoke per 0.4 second time interval and its distance from the source.

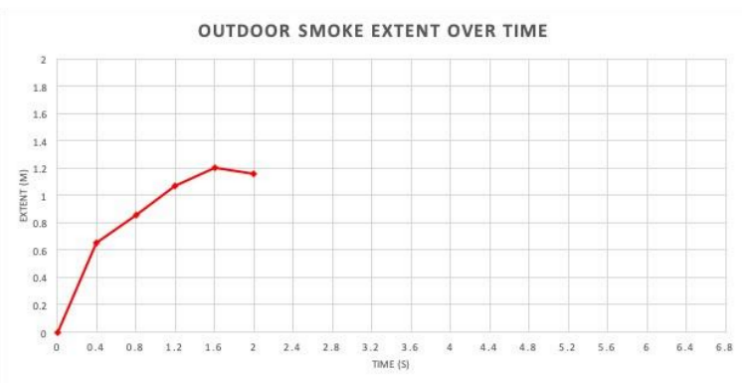

Figure 15. 2D Graph of Cigarette Smoke Extent vs Time in an Outdoor Environment

Figure 16 shows a 3D graph of the cigarette smoke percent density over time accounting the smoke's relative extent from the source in an outdoor environment. This can be helpful in an in-depth analysis of the resulting graphs. The first figure below shows the relationship of time, percent density, and relative distance covered of the cigarette smoke. The second figure shows that the density of the cigarette smoke also increased with its distance until it reached a certain peak and decreased until it totally diminished. The third figure visibly shows the unusual movement of smoke based on the output video of the code in which the extent from the time interval of 1.6 seconds to 2.0 seconds decreases that deviate from what was expected.

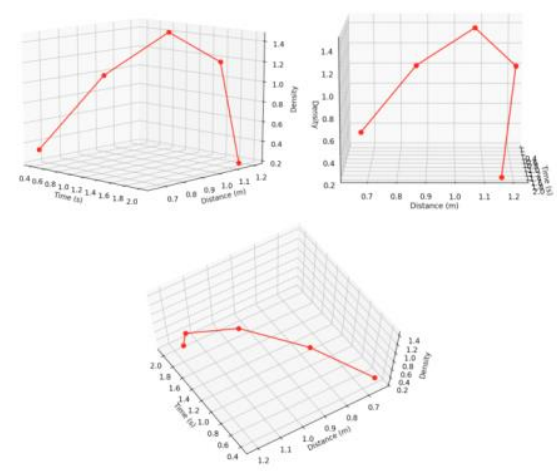

Figure 16. 3D Graph of Cigarette Smoke Density vs Time vs Extent Relative from the Source in an Indoor Environment

\subsection{Comparison of the Results from the Two Environments}

Comparing the two environment, the time difference of 4 seconds equates to how fast the smoke dispersed and clearly the cigarette smoke dispersed faster in an outdoor environment due to the external factor contributed by wind and such, the resulting cigarette smoke stayed longer on the indoor environment and was more evidently visible for a longer time compared to the outdoor. Figure 17 and Figure 18 shows the evident visualization of this difference in time duration of two different environments.

Another evident difference from the two environments shown in Figure 17 was that the smoke percent density for the outdoor environment was relatively higher than that in an indoor environment. Wind effect was, again, one of the main reasons for this big difference in values of percent density. The presence of wind can increase turbulence which then widens the area occupied by the smoke in an environment hence the increase in the value of percent density in the 
outdoor environment where the wind affected the set-up more than the isolated set-up for the indoor environment.

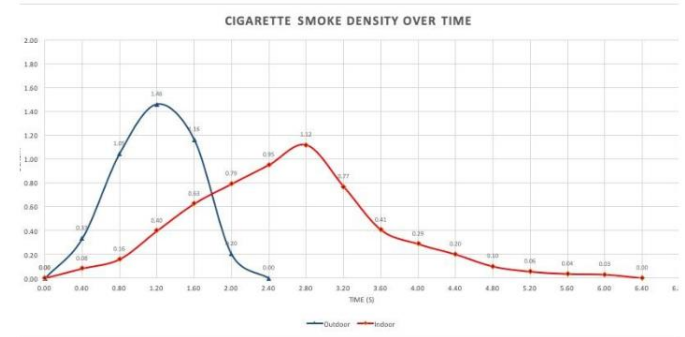

Figure 17. 2D Graph of Cigarette Smoke Density vs Time in an Outdoor Environment and Indoor Environment for Comparison

Figure 18 shows a combined graphs of cigarette smoke extent over time for both indoor and outdoor environments. Previously discussed, these two graphs both exhibits a spike or unexpected movement towards the final seconds. This was caused by the limitation of the program generated. Towards the end, the transparency of the smoke became apparent and so appeared to be not that visible in images. Hence, the problem in detection towards the end. The jump in values can also be caused by the false detection of smoke towards the end because of transparency. The system may have detected two or more different zones of smoke pixels that may come from the same puff that caused the values to jump towards the end. Another was that the acquisition of the coordinates for the maximum extent, extracted an $(\mathrm{x}, \mathrm{y})$ pixel coordinates yet the analysis stuck to $\mathrm{x}$ coordinates only, because the resulting data for the Y coordinate were not logical due to the detection was not particle based. This being said, the smoke detection was optically based, thus it will only detect the moving puff and not a single particle. The resulting y coordinates did not reflect the overall movement of the smoke, but it only signifies the location of the detected region.

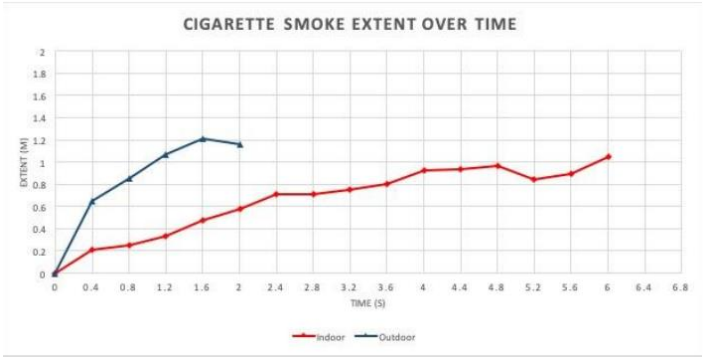

Figure 18. 2D Graph of Cigarette Smoke Extent vs Time in an Outdoor Environment and Indoor Environment for Comparison

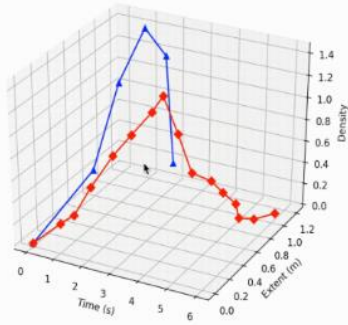

Figure 19. 3D Graph of Cigarette Smoke Density vs Time vs Extent Relative from the Source for Both Indoor and Outdoor Environment

\section{CONCLUSION}

\subsection{Conclusion}

The implementation of specific methods of smoke detection by applying different functions such as image subtraction, morphological filter, thresholding, contouring, and masking enables this study to create a program designed for optical RGB cameras in visualizing cigarette smoke dispersion. The researchers were able to utilize an optical RGB camera and digital image processing techniques in determining cigarette smoke dispersion. The spatiotemporal visualization for the indoor and outdoor environment, through the series of processes that were created in PyCharm, highlighted the smoke dispersion. The results shown quantitatively realistic and relatively accurate dispersion of cigarette smoke.

Based on the quantitative analyses of the smoke dispersion, relative smoke density and relative maximum horizontal extent over an elapsed time have significant differences in each environment. For the indoor environment, the smoke peaked its percent density at 2.80 seconds from it was puffed from the source and achieved a percent density of $1.119 \%$ while its distance or extent reached a maximum distance of 1.048 meters for the visible smoke. For the outdoor environment, the smoke peaked its percent density at 1.2 seconds from it was puffed from the source and achieved a percent density of $1.46 \%$ while its distance or extent reached a maximum distance of 1.21 meters for the visible smoke. The study was able to extract a relative behavior of the cigarette smoke in terms of its maximum extent and smoke density. Overall, the study was able to create a program designed for optical RGB cameras for specifically, cigarette smoke detection and for the analysis of its dispersion through the use of photogrammetric software and various libraries for digital image processing. This, in turn, can lead to a costefficient way of understanding air pollutant transport and management with the use of close-range photogrammetry.

\subsection{Recommendations}

The created program was specifically designed for cigarette smoke and was able to detect and mask the white smoke. The flexibility of the program was barely tested with different smoke sources and it is recommended that detection of black smoke from factories, fire and haze detection are to be explored. The study could be extended by modifying the methodology through various camera set-ups for observing smoke dispersion in different perspectives. With that modification, the study can be used in $3 \mathrm{D}$ visualization and analysis. To further improve the buffer zones for smoking area, the methodology of this study should account for moving or dynamic sources of smoke in all directions. The process in obtaining the percent density and maximum extent was relative because the frames were not georeferenced thus the resulting data did not give the exact location of smoke. To georeference the image, known coordinates of objects present in the image should be known in order to acquire the exact location of the smoke. Stationary devices like CCTV can be easily used to study and monitor smoke dispersion in different location. In that case, methodology will be refined by studying different camera angles and infusing these different videos to create a $3 \mathrm{D}$ version of smoke dispersion that would give the parameters of smoke such as the extent, height, and other extractable parameters. The acquired relative smoke density can further be improved with the use 
of specific sensors to come up with the value of smoke concentration that could be useful in air quality assessment and air quality management. The study can be replicated in larger area with multiple smoke sources. The methods are the same but different set-ups are needed. Multiple cameras are needed to capture all the existing smoke in the area.

\section{REFERENCES}

Baumbach, G. (2014). Air quality control formation and sources, dispersion, characteristics and impact of air pollutants - measuring methods, techniques for reduction of emissions and regulations for air quality control. Berlin: Springer.

Çelik T., Özkaramanl H., Demirel H., (2007). Fire and Smoke Detection Without Sensors: Image Processing Based Approach. 15th European Signal Processing Conference. Electrical and Electronic Engineering, Eastern Mediterranean University.

Deldjoo Y., Nazary F., Fotouhi A.M., (2015). A Novel Fuzzy-Based Smoke Detection System Using Dynamic and Static Smoke Features. Iranian Conference on Electrical Engineering.

Haupt S., Long K., Hendrickson M., Keay J., (2014). Applying Photogrammetric Techniques to Study Smoke Plumes. Accessed September 1, 2018. https://www. researchgate.net/publication /267226969

Hwang U., Jeong J. (2016). Computer Vision Based Smoke Detection Method By Using Colour and Object Tracking. Department of Electronic and Computer Engineering.

Lorenz, E.N., (1963) Deterministic Nonperiodic Flow, J. Atmos. Sci., 20, 130-141.

Lu B., Wang J., Ma X., Lu H., (2018). Air Pollution Forecasts: An Overview. Int. J. Environ. Res. Public Health 2018, 15, 780; doi:10.3390/ijerph15040780

Mutar A.F., Dway G.H. (2018). Smoke Detection Based on Image Processing by Using Grey and Transparency Features. Journal of Theoretical and Applied Information Technology.

Salmon, J. (2014). State of: Close-Range Photogrammetry. Magazine. https://www.xyht.com/lidarimaging/state-ofclose-range-photogrammetry/. Accessed May 19, 2019.

Wang Y., Chua T., Chang R., Pham N., (2012). Real-Time Smoke Detection Using Texture and Color Features. Institute for Infocomm Research, Agency for Science, Technology and Research, Singapore. https://www.researchgate.net /publication/242401991

Wyngaard, J.C. (2010) Turbulence in the Atmosphere, Cambridge University Press, 408 pp.

Visscher, A. D. (2014). Air dispersion modeling: Foundations and applications. Hoboken: Wiley. 